\title{
Fast Surface Crystal Growth on Organic Glasses Studied by High - Resolution Microscopy
}

\author{
Mariko Hasebe, Daniele Musumeci, and Lian Yu
}

School of Pharmacy University of Wisconsin - Madison, Madison, WI 53705, USA

Amorphous solids (glasses) are of great interest due to many useful properties they possess. While better known amorphous materials are inorganic such as windows, there is a strong interest in developing organic or molecular glasses as photo - and electro - active materials [1], [2], matrices for preserving proteins and organism [3], and vehicles for delivering poorly soluble drugs [4]. For example, amorphous solids are generally more soluble and faster dissolving than their crystalline counterparts; a property useful for delivering drugs whose bioavailability is limited by their poor solubility. Recently, this strategy has been used to reformulate the AIDS drug Kaletra to reduce the number of pills taken by the patient and eliminate the need for refrigeration, which is a major advantage for patients in rural and underdeveloped areas such as Africa. Unfortunately, few amorphous products are available nowadays, due to the fact that it is not well established to understand the stability of organic glasses against crystallization. For an amorphous material, stability against crystallization is essential because crystallization negates its advantages.

Recent studies have shown that organic glasses exhibit substantially faster crystal growth at the free surface than in the interior [5], [6], [7], [8]. The phenomenon is relevant to the fundamental understanding of crystallization because it is apparently uncommon for other classes of materials. To understand this fast mode of crystal growth, we studied the surface crystallization of indomethacin (IMC) glasses in two polymorphic modifications ( $\alpha$ and $\gamma$ ) by observation through a light microscope. The surface crystal growth rate of $\alpha$ IMC was terminated by heating above $T_{\mathrm{g}}$, while the surface crystal growth rate of $\gamma$ IMC is little affected.

We used high-resolution microscope such as SEM and AFM in order to understand the mechanism of material transport associated with the fast crystal growth at the surface. AFM and SEM measurements revealed both of polymorphs in IMC have growth fronts substantially higher than the glass surface at $30^{\circ} \mathrm{C}\left(T_{\mathrm{g}}-12^{\circ} \mathrm{C}\right)$ in Figure 1 and 2 . Further more, they showed a well-defined depletion zones around growing crystals in two polymorph of IMC, which suggested surface diffusion supplied material from the glass to the crystal. SEM images of $\alpha$ IMC surface crystals in cross-sectional view demonstrated that the depletion zone was filled in, and the surface crystals were wetted and eventually buried by activated liquid flow above $T_{\mathrm{g}}$. Conversely, $\gamma$ IMC still showed the depletion zone and the surface crystal layer became wavy, but it was not buried above $T_{\mathrm{g}}$.

We conclude that the upward growth of crystal at the surface of $\alpha$ IMC is no longer sustained above $T_{\mathrm{g}}$ because the surface crystals are embedded in activated liquid flow. This different behavior in two polymorphic modifications is explained by the fiber-like crystals of $\alpha$ IMC being more vulnerable to the activated liquid flow than the plate-like $\gamma$ IMC crystals. Our finding by high -resolution and real time measurements of crystal growth at the surface enables to explain recently discovered surface phenomena of organic glasses and to understand the fast surface mobility. 


\section{Reference:}

[1] Shirota Y., J Mater Chem 15 (2005), p.75-93

[2] De Silva, A., Felix N.M., and Ober C.K., Adv Mater 20 (2008), p.3355-61

[3] Crowe, J. H, Carpenter, J. F., and Crowe, J.H., Annu. Rev. Physiol 60 (1998), p.73-103.

[4] Yu, L., Adv. Drug Delivery Rev 48 (2001), p.27-42.

[5] Wu, T., and Yu, L., Pharm. Res 23 (2006), p.2350-2355

[6] Zhu, L., Wong, L., Yu, L., Mol. Pharm 5 (2008), p.921-926

[7] Wu, T. et al., Langmuir 23 (2007), p.5148-53

[8] Sun, Y. et al., Proc Natl Acad Sci USA 108 (2011), p.5990-5995

[9] The authors acknowledge funding from the National Science Foundation (DMR-1206724) for supporting this work
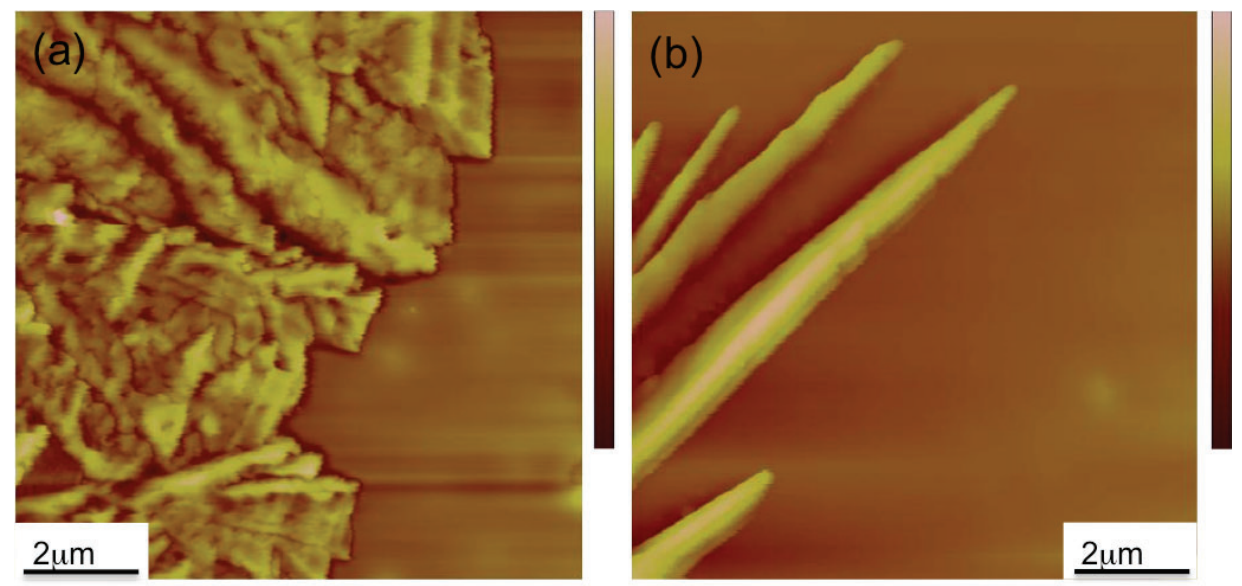

Figure 1. AFM images of $\gamma \operatorname{IMC}(\mathrm{a})$ and $\alpha \operatorname{IMC}(\mathrm{b})$ at $40^{\circ} \mathrm{C}\left(T_{\mathrm{g}}-2^{\circ} \mathrm{C}\right)$. The color scale is $300 \mathrm{~nm}(\mathrm{a})$ and $1.5 \mu \mathrm{m}(\mathrm{b})$

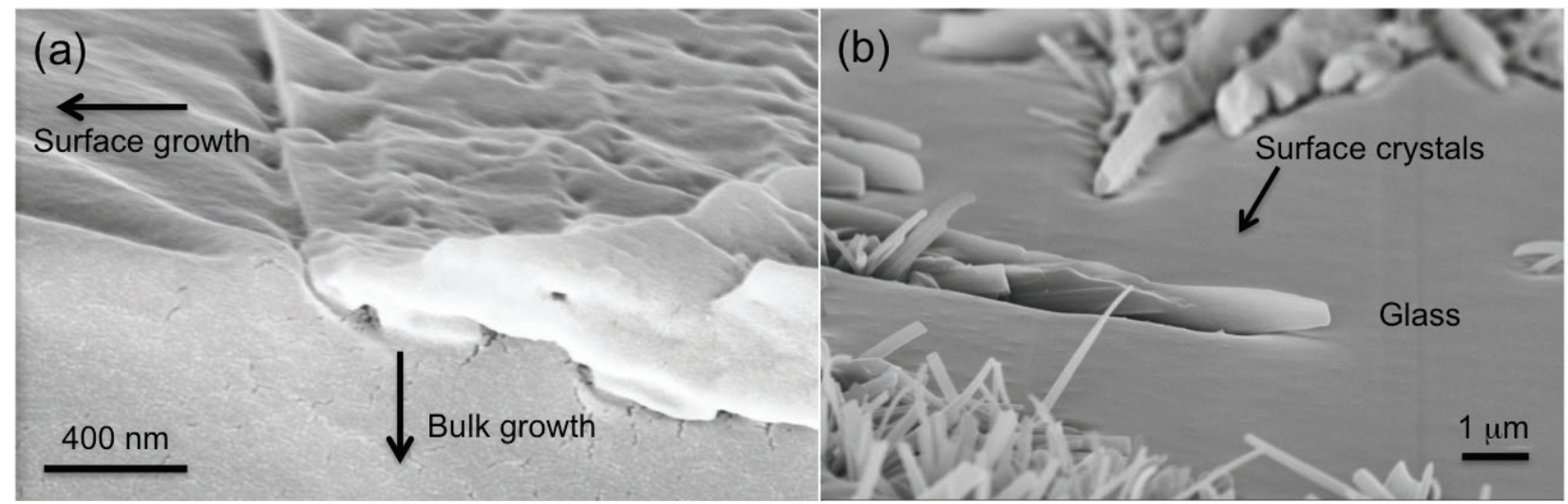

Figure 2. SEM images in cross-sectional view of $\gamma$ IMC (a) and top view of $\alpha$ IMC (b) surface crystals on IMC glass at $30^{\circ} \mathrm{C}\left(T_{\mathrm{g}}-12^{\circ} \mathrm{C}\right)$ 